\title{
Estrogènes et spermatozoïdes
}

\author{
I.GALERAUD-DENIS*, E.MARIE* et S.CARREAU \\ *Clinique de Reproduction Humaine, CHRU Clémenceau, Caen \\ et Laboratoire de Biochimie-IRBA, UPRES EA 2608, Université, 14032-CAEN Cédex.
}

\section{RESUME}

Les estrogènes sont depuis longtemps considérés comme des hormones sexuelles typiquement femelles; cependant ils semblent jouer un rôle éminent dans le testicule tout aussi important que leur fonction endocrine classique. L'aromatase (enzyme qui transforme irréversiblement les androgènes en estrogènes) est impliquée notamment dans le développement, la différenciation sexuelle et le comportement, la reproduction, les métabolismes osseux et lipidique, le fonctionnement du cerveau; son importance est donc capitale chez l'homme. Les données mettant en évidence au sein du testicule plusieurs types cellulaires opérationnels dans la production d'estrogènes, associés à la présence de récepteurs spécifiques (ER $\alpha$ et ERß) à plusieurs niveaux du tractus génital mais aussi sur certaines cellules sexuelles, plaident donc fortement en faveur d'un rôle intrinsèque de ces hormones femelles en dehors de leur impact connu sur la secrétion gonadotrope. Un certain nombre de preuves et d'arguments permettent de penser qu'une (ou des) relation (s) existe (nt) entre estrogènes et infertilité, en particulier au niveau épididymaire où la composition du fluide luminal de la tête de l'épididyme (zone essentielle pour la maturation du spermatozoïde) serait modulée par ces hormones femelles. Chez l'homme, les sites de production et le rôle des estrogènes dans la spermatogenèse et la spermiogenèse ne sont pas encore totalement élucidés; cependant, dans l'étude des mécanismes physiopathologiques impliqués dans l'infertilité la participation des estrogènes est de plus en plus envisagée. En conséquence, les estro- gènes se révèlent donc être essentiels pour la reproduction du mâle ce qui nous amènent à reconsidérer certaines notions de la physiologie de la reproduction masculine, avec non seulement des régulations androgéno-dépendantes mais aussi estrogéno-contrôlées.

Mots-clés : Estrogènes, spermatogenèse, spermatozoïde, aromatase, fertilité, homme

\section{INTRODUCTION}

Les testicules de mammifères ont une double fonction : production de gamètes et synthèse de stéroïdes qui sont notamment sous le contrôle des gonadotropines FSH et LH, dont l'action est modulée localement par de nombreuses substances produites par les cellules gonadiques elles-mêmes [9, 71]. Parmi ces régulateurs paracrines et autocrines, les hormones stéroïdes, androgènes et estrogènes, jouent un rôle éminent $[12,13,36,74]$. Les estrogènes sont depuis longtemps considérés comme des hormones sexuelles de type "femelle" alors que la testostérone, chef de file des androgènes, est l'apanage du mâle. Cependant l'existence et le rôle de ces hormones "femelles" chez le mâle suscitent aujourd'hui beaucoup de débats et mises au point [5,

Correspondance: Dr S.CARREAU

Tél: 0231565488 - Fax : 0231565320

E-mail: carreau@ibba.unicaen.fr 
73,74]. Si on reprend l'historique de la découverte des estrogènes, ils furent d'abord mis en évidence dans l'urine de l'étalon par Zondek en 1934 [91]; puis dans le testicule des mammifères la synthèse fut démontrée in vitro et in vivo à partir des années 50 [29, 41, 47, 70]. En conséquence il n'est pas surprenant que le rete testis de rat renferme des concentrations d'estrogènes comparables, voire supérieures à celles observées dans le sérum de la femelle $[27,46]$. Il en est de même chez l'homme où la concentration en estrogènes est plus élevée dans le fluide du rete testis que dans la circulation périphérique [33].

Dans cette mise au point relative au rôle des estrogènes au niveau des fonctions de reproduction du mâle, nous nous sommes plus particulièrement centrés sur les points suivants: 1) le spermatozoïde constitue-t-il un site de synthèse des estrogènes et 2) représente-t-il une cellule-cible pour les hormones femelles?

La conversion irréversible des androgènes en estrogènes est assurée par l'aromatase. Cette enzyme est composée d'un cytochrome P450 aromatase spécifique et d'une flavoprotéine ubiquitaire, la NADPH cytochrome P450 réductase. Contrairement à la localisation non spécifique du complexe (cerveau, tissu adipeux, muscle, gonades, placenta, surrénales, peau, os,...), le gène CYP 19 codant pour l'aromatase (chromosome 15 q21.1) est exprimé dans un nombre limité de tissus en raison de l'existence de promoteurs spécifiques $[75,76]$. L'intervention de facteurs modulant l'expression du gène CYP 19 serait donc déterminante dans le maintien de l'équilibre androgènes/ estrogènes intratesticulaire. Au niveau de la gonade mâle, l'aromatase a été immunolocalisée dans les cellules de Leydig de nombreux mammifères ; cependant chez les rongeurs, l'activité aromatase est présente non seulement dans les cellules de Leydig mais aussi dans les cellules de Sertoli et, fait nouveau, dans les cellules germinales (l'activité est 4 à 5 fois plus forte dans les spermatozoïdes que dans les spermatocytes [11-13, 40, 50]. En utilisant des sondes moléculaires spécifiques, la présence d'ARN messager de l'aromatase a été démontrée dans les cellules somatiques et les cellules germinales en particulier chez les ron- geurs $[39,40,49-51,64]$ et les ursidés [86]. L'existence de la protéine dans les cellules germinales haploïdes, notamment les spermatides allongées et les spermatozoïdes [34, 50], est corroborée par les travaux de Janulis et al. $[39,40]$ montrant que cette activité enzymatique diminue au cours du transit épididymaire et suggérant que celle-ci serait donc associée à la perte de la goutte cytoplasmique dans le corps et la queue de l'épididyme. Ces données apportent donc une preuve irréfutable d'une source additionnelle d'estrogènes en dehors des cellules de Leydig dans le testicule des mammifères.

Quant à l'action des estrogènes sur le spermatozoïde, elle pourrait être médiée par un ou deux types de récepteurs ( $\mathrm{ER} \alpha$ et $\mathrm{ER} \beta$; [43]) dont l'identité est à confirmer et les rôles pas encore bien précisés en termes de cellules et gènes cibles (tableau 1). Le rôle de l'estradiol chez le mâle n'est sans doute pas uniquement localisé aux régions post-testiculaires puisque la prolifération des gonocytes [52] et la synthèse des cadhérines par exemple sont dépendantes de l'estradiol, observations qui sont validées par la présence d' ERß dans pratiquement toutes les cellules germinales chez le rat $[72,88]$. Au cours de la spermatogénèse, l'ARNm codant pour ERß et la protéine ERß sont nettement exprimés depuis le stade pachytène jusqu'au stade spermatide ronde $[21,88]$; il en est de même pour l'ARNm codant pour l'aromatase $[50,64]$. En conséquence, le décalage temporel au cours de la spermatogenèse entre l'expression des ARNm codant pour l'aromatase et l'apparition d'une aromatase active favoriserait un rapport androgènes / estrogènes plutôt en faveur de la testostérone à des étapes androgéno-dépendantes tels les spermatocytes pachytènes et les spermatides rondes $[59,89]$.

Les estrogènes interviendraient aussi dans la régulation de la spermiogénèse. En effet, la maturation des spermatides chez le rat est réduite en présence d'inhibiteurs de l'aromatase [87] et de tamoxifène [28]. Shetty et al. $[77,78]$ ont confirmé ces résultats et montré une réduction du nombre de spermatides allongées et de leur qualité après l'administration d'antiestrogènes ou d'inhibiteurs de l'aromata- 


\begin{tabular}{lccc}
\hline Tissu/Cellules & ER $\alpha$ & ERß & Aromatase \\
\hline Leydig & + & $+/-$ & + \\
Peritubulaire & - & + & $+/-$ \\
Sertoli & - & + & $\mathbf{N D}$ \\
Spermatogonie & - & + & + \\
Spermatocyte pachytène & - & + & + \\
Spermatide ronde/allongée & - & $+?$ & $\mathbf{+}$ \\
Spermatozoïde & $+?$ & $\mathbf{N D}$ & $\mathbf{N D}$ \\
Rete testis & + & $+/-$ & $\mathbf{N D}$ \\
Canaux efferents & + & + & $\mathbf{N D}$ \\
Epididyme & $+/-$ & + & $\mathbf{N D}$ \\
Vésicules séminales & - & & \\
Prostate & - & & \\
\end{tabular}

ND: non étudiée

+ ?: récepteur non typé $\alpha$ ou $\beta$

se chez le rat comme chez le singe.

Des récepteurs aux estrogènes de type $\alpha$ et $\beta$ ont été localisés au niveau des canaux efférents, site de réabsorption du fluide testiculaire : $E R \alpha$ chez le rat et l'ouistiti [17, 24, 35] et ERß [72]. La présence de récepteurs aux estrogènes de type $\alpha$ est plus controversée au niveau de l'épididyme mais les ERß sont présents [24, 35, 43, 84]. D'autres informations permettent de penser qu'il existe une relation entre estradiol et maturation épididymaire: l'estradiol pourrait notamment agir au niveau de la réabsorption du fluide testiculaire dans les canaux efférents. En effet, les bases biochimiques de la réabsorption font appel à la présence de canaux ioniques (canal $\mathrm{Na}+/ \mathrm{K}+$ /ATPase, canal chlore) et canaux aqueux. L'aquaporine 1 pourrait ainsi être impliquée dans les phénomènes de réabsorption du fluide au niveau des canaux efférents chez le rat [25] et cette protéine serait régulée par les estrogènes. La composition du liquide épididymaire qui varie de la tête à la queue est non seulement androgéno-dépendante mais pourrait être également influencée par les estrogènes [57]. L'injection intramusculaire d'estrogènes chez des rats adultes induit une augmentation des concentrations en sodium et chlorures et diminue celles de calcium et phosphates [42]. Un traîtement prolongé par du tamoxifène [4] entraine une augmentation de la liaison de la $\beta$-galactosidase sur les spermatozoïdes et une diminution de celle de l' $\alpha$-mannosidase. Le tamoxifêne pourrait agir directement sur les récepteurs aux estrogènes ou comme inhibiteur du canal $\mathrm{Na}+/ \mathrm{K}+/$ ATPase [69]; les modifications en résultant pouvant affecter partiellement le processus de maturation des spermatozoïdes, en particulier la non acquisition de la mobilité et la perte de capacité de fécondance [28].

Ces observations plaident fortement en faveur d'un rôle des estrogènes à plusieurs niveaux dans le tractus génital du mâle et en particulier au cours de la maturation épididymaire des spermatozoïdes. De fait il y a un parallélisme entre l'activité aromatase du spermatozoïde qui décroît très rapidement au cours du transit épididymaire, et la présence d'ER $\alpha$ / ERß en particulier dans les canaux efférents et la tête proximale de cet organe. Ainsi, la création de modèles animaux tels que les souris ERKO (extinction du gène codant pour $E R \alpha$ ) entraine l'apparition à partir du 20ème jour d'une dilatation des tubes séminifères et des canaux efférents, une numération en spermatozoïdes épididymaires réduite, une motilité 
des spermatozoïdes altérée et une inefficacité des spermatozoïdes à féconder des ovocytes de souris $[20,53]$. Néanmoins dans un récent travail publié par le groupe de Simpson [23], il semblerait que des souris mâles déficientes en aromatase (ArKO) soient capables de se développer normalement et procréeer (la descendance est assurée); il convient toutefois de signaler qu'après 11 semaines d'âge (comme pour les mâles ERKO), les males dépourvus d'aromatase deviennent progressivement infertiles.

\section{QU'EN EST-IL CHEZ L'HOMME?}

Le rôle des estrogènes chez le mâle a récemment été réexaminé en raison

1) des découvertes chez l'homme de l'importance des estrogènes dans le développement du tractus génital et la fertilité $[8,60,79]$,

2) des rapports faisant état d'un déclin de la spermatogenèse $[2,85]$ et

3) de l'incidence des xénobiotiques [18] sur les fonctions sexuelles de l'homme. Les observations cliniques ont apporté de précieuses informations quant aux rôles des hormones femelles chez le mâle. Quelques cas de déficit en aromatase par mutation sur le gène CYP 19 ont été publiés $[6,8,60]$ avec des troubles de la croissance (homme de très grande taille), un hyperinsulinisme et une perturbation du bilan lipidique. Les concentrations en gonadotrophines sont très élevées alors que celles de l'estrone et de l'estradiol sont indétectables, démontrant de fait un rôle des estrogènes dans le feed-back négatif hypophysaire. Aucun spermogramme n'a pu être étudié $[6,60]$. Les observations sont donc peu documentées quant à l'impact du déficit en aromatase sur la fertilité des patients; seul Carani et al [8] ont fait état d'un tableau d'infertilité masculine avec la présence de petits testicules et d'une oligoasthénospermie très sévère ( 1 million de spermatozoïdes immobiles / $\mathrm{ml}$ ) chez un patient présentant une mutation homozygote inactivant le gène de l'aromatase.

A l'inverse, des concentrations supra-physiologiques d'estrogènes provoquent aussi des anomalies de la spermatogenèse et inhibent la fertilité $[7,55,56,61,63,65,90]$. Cependant si les taux d'estrogènes sont trop élevés, la découverte récente dans le testicule des rongeurs et de l'homme d'une estrogène sulfotransferase leydigienne permettrait alors de diminuer le taux d'estrogènes actifs en les sulfatant [80]. Quant aux récepteurs des estrogènes une seule pathologie est décrite : il s'agit d'une mutation portant sur le gène où l'exon 2 présente un codon stop en 157 [79]. L'étude du spermogramme a révélé une numération en spermatozoïdes correcte associée à une viabilité réduite. Tous ces faits sont à rapprocher de travaux plus anciens rapportant un déficit stéroïdogène et notamment en estrogènes dans le plasma séminal d'hommes azoospermes [68, 83].

Les observations que nous venons de décrire nous amènent à faire le point sur les sites de production des estrogènes et leurs cibles dans le tractus génital humain. Outre les cellules de Leydig et de Sertoli [10, 26, 38, 48, 66, 67] , une activité aromatasique a pu être mise en évidence dans des cellules germinales humaines après transformation maligne [65]. La capacité du spermatozoïde humain à métaboliser les stéroïdes a été décrite [30,31]. Ces résultats sont en accord avec des données antérieures [16] rapportant des concentrations endogènes faibles mais mesurables de progestérone, testostérone, et estradiol-17ß dans les spermatozoïdes. Un récepteur aux estrogènes de type $\beta$ (ERß) a été cloné dans le testicule humain [44, 62]. En outre, le groupe de Gustafsson a finement localisé par hybridation in situ cet ERß notamment dans les spermatides rondes et à un degré moindre dans les spermatocytes pachytènes [21]; aucun signal n'a été détecté dans les cellules somatiques. Dans les spermatozoïdes humains un récepteur (ARNm et protéine) aux estrogènes vient d'être mis en évidence [19]. Signalons aussi la présence de récepteurs pour les estrogènes et la progestérone dans les cellules des canaux efférents chez l'homme [22].

Toutes ces informations nous amènent à évoquer les travaux antérieurs de Beck et al. [3] et de Cheng et Boettcher [15] suggérant que l'addition d'estradiol à une suspension de spermatozoïdes humains stimule leur mobilité. Par ailleurs, le groupe de Czyba [37] a confirmé ces observations et démontré que des spermato- 
zoïdes provenant de patients asthénospermiques incubés en présence d'estradiol deviennent plus mobiles; en outre, leur métabolisme oxydatif, leur survie et la pénétration ovocytaire sont optimisés. En présence de citrate de clomiphène et de tamoxifène, les effets précédemment décrits sont abaissés [14]. Tous ces éléments sont en faveur d'un rôle des estrogènes dans l'évolution finale du spermatozoïde, rôle concevable compte tenu de l'environnement hormonal dans lequel les spermatozoïdes vont baigner dans le tractus génital femelle. L'estradiol secrété pourrait alors agir comme un agent chimiotactique sur les spermatozoïdes et reconnaître les récepteurs membranaires du spermatozoïde (Baldi et al; 1999; communication personnelle). Ces récepteurs seraient associés à un système de seconds messagers à réponse rapide tels que l'AMP cyclique ou le système calcium-calmoduline dont on connaît leurs implications dans la mobilité du sperme [81, 82]. Ces données sont donc en faveur d'effets non-génomiques des stéroïdes, d'ailleurs confirmés par l'existence de récepteurs membranaires pour la progestérone sur le spermatozoïde humain [54] ce qui permettrait l'expression de la progestérone dans l'induction de la réaction acrosomique.

Compte tenu des données publiées sur la baisse de la concentration et la diminution de la qualité des spermatozoïdes humains [2], les xénoestrogènes ont été évoqués [32, 85]. Ces derniers peuvent interagir non seulement avec les protéines de transport des stéroïdes mais aussi avec les récepteurs des androgènes [18], les récepteurs ER $\alpha$ et/ou ERß et stimuler l'activité transcriptionnelle de ces récepteurs [45]. De plus, on sait que l'exposition prénatale aux estrogènes provoque des anomalies du sperme et du tractus génital [1].

Cependant il convient de rappeler que le blocage de la synthèse d'estrogènes (administration d'inhibiteur de l'aromatase) chez les singes (Macaca radiata) entraine une interruption de la maturation des cellules germinales (diminution du nombre des spermatides) et donc une réduction $(90 \%)$ de la production des spermatozoïdes associée avec une chute de la motilité $[77,78]$; ce qui indéniablement plaide en faveur du rôle positif des estrogènes dans la spermiogenèse des primates.

En conséquence, compte tenu de la présence de l'aromatase dans les cellules gonadiques, on assiste à des modifications du rapport androgènes / estrogènes. Si la testostérone est le médiateur principal du développement testiculaire, les estrogènes produits localement sont sans aucun doute impliqués dans le contrôle de la stéroïdogenèse, mais aussi dans la spermatogenèse et la spermiogenèse à plusieurs niveaux. On peut rappeler cette réflexion récente de Sharpe [73] qui dit: "les testicules comptent sur les hormones femelles". Aujourd'hui nous manquons de connaissances sur les gènes cibles estrogèno-dépendants, ce qui aiderait à mieux appréhender le rôle positif (ou négatif) de ces hormones femelles et donc permettrait de développer de nouvelles approches thérapeutiques (ou contraceptives) en utilisant par exemple des antagonistes ou des agonistes de l'aromatase. En somme, une étude approfondie quant à la participation des estrogènes dans les processus de maturation des spermatozoïdes chez l'homme s'avère indispensable. Les données récemment acquises sur les nouveaux sites de synthèse des estrogènes dans les cellules testiculaires et celles relatives à la présence des récepteurs (ERB) nous amènent à proposer une relecture de la physiologie testiculaire non seulement androgénodépendante mais aussi estrogéno contrôlée, en particulier chez l'homme.

Remerciements : Les auteurs remerciement particulièrement leurs collaborateurs et les laboratoires Organon pour leur soutien financier.

\section{REFERENCES}

1. ARAI Y., MORI T., SUZUKI Y., BERN H.A. Longterm effects of perinatal exposure to sex steroids and diethylstilbestrol on the reproductive system of male mammals. Int.Rev.Cytol., 1983; 84 : 235-268.

2. AUGER J., KUNSTMANN J.M., CZYGLIK F, JOUANNET P. Decline in semen quality among fertile men in Paris during the past 20 years. $\mathrm{N}$. Engl. J. Med., 1995; 332 : 281-285.

3. BECK K.J., HERSCHEL S., HUNGERSHOFER R., SCHWINGER E. The effect of steroid hormones on motility and selective migration of $\mathrm{X}$-and Y-bearing 
human spermatozoa. Fertil. Steril., 1976;27:407412.

4. BELMONTE S., MATURANO M., BERTINI F., PUSIOL E., SARTOR T., SOSA A.

Changes in the content of rat epididymal fluid induced by prolonged treatment by tamoxifen. Andrologia, 1998; $30: 345-350$.

5. BERGH J.P. Oestrogens-essential for reproduction in males, too. Eur.J.Endocrinol., 1998, 138 : 497-498.

6. BULUN S.E. Aromatase deficiency in women and men : would you have predicted the phenotypes? J. Clin. Endocrinol. Metab., 1996; 81 : 867-71.

7. BULUN S.E., NOBLE L.S., TAKAYAMA K. et al. Endocrine disorders associated with inappropriated high aromatase expression. J. Steroid. Biochem. Molec.Biol., 1997; 61: 133-139.

8. CARANI C., QIN K., SIMONI M., et al. Effect of testosterone and estradiol in a man with aromatase deficiency. N. Engl.J. Med., 1997; 337 : 91-95.

9. CARREAU S. Germ cells-Sertoli cells interactions and Leydig cell function. In: Dufau M . L . , Fabbri A., Isidori A., eds. Cell and Molecular Biology of the testis. Frontiers in Endocrinology 1994; $5: 137-48$.

10. CARREAU S. Paracrine control of human Leydig cell and Sertoli cell functions. Folia Histochem. Cytobiol. 1996; 34 : 111-9.

11. CARREAU S ., LEVALLET J. L'aromatase testiculaire: du gène à la protéine. Andrologie, 1998; 8 : 191-198.

12. CARREAU S., BILINSKA B., LEVALLET J. Les cellules germinales mâles : une source nouvelle d'estrogènes dans le testicule des mammifêres. Ann. Endocrinol., 1998b; 59 : 79-92.

13. CARREAU S., GENISSEL C., BILINSKA B., LEVALLET J. The oestrogen sources in the testis and the reproductive tract of the male. Int. J. Androl., sous presse, 1999.

14. CHAN S.Y.W., WANG C.C.L., TANG L.C.H. Effect of clomiphene citrate on human spermatozoa motility and fertilizing capacity in vitro. Fertil .Steril., 1985; $43: 773-776$.

15. CHENG C.Y., BOETTCHER B. The effect of steroids on the in vitro migration of washed human spermatozoa in modified tyrode's solution or in fasting human blood serum. Fertil. Steril., 1979; 32 : 566-570.

16. CHEW P.C.T., LOGANATH A., PEH K.L., CHOW W.P., GUNASEGARAM R., RATNAM S.S. Concentrations of intracellular sex steroids in human spermatozoa. Arch. Androl., 1993; 30 : 165-170.

17. COOKE P.S., YOUNG P., HESS R.A., CUNHA G.R. Estrogen receptor expression in developing epididy. mis, efferent ductules, and other male reproductive organs. Endocrinology, 1991; 128 : 2874-2879.
18. DANZO B.J. Environmental xenobiotics may disrupt normal endocrine function by interfering with the binding of physiological ligands to steroid receptors and binding proteins. Environ. Health Perspect., 1997; $105: 294-301$.

19. DURKEE T.J., MUELLER M., ZINAMAN M. Identification of estrogen receptor protein and messenger ribonucleic acid in human spermatozoa. Am. J. Obstet. Gynecol., 1998; 178 : 1288-1297.

20. EDDY E.M. WASHBURN T.F., BUNCH D.O. et al. Targeted disruption of the estrogen receptor gene in male mice causes alteration of spermatogenesis and infertility. Endocrinology $1996 ; 137: 4796-805$.

21. ENMARK E., PELTO-HUIKKO M., GRANDIEN K. et al. Human estrogen receptor- $\beta$ gene structure, chromosomal localization, and expression pattern. J.Clin.Endocrinol.Metab., 1997; 82 : 4258-4265.

22. ERGUN S., UNGERFROREN H., HOLSTEIN A.F., DAVIDOFF M.S. Estrogen and progesterone receptors and estrogen receptor-related antigen (ER-D5) in human epididymis. Molec. Reprod. Dev., 1997; $47: 448-455$.

23. FISHER C.R., GRAVES K.H., PARLOW A.F., SIMPSON E.R. Characterization of mice deficient in aromatase (ArKo) because of targeted disruption of the cyp 19 gene. Proc. Natl. Acad. Sci. (USA) 1998; 95 : 6965-6970.

24. FISHER J.S., MILLAR M.R., MADJIC G., SAUNDERS P.T.K., FRASER H.M., SHARPE R.M. Immunolocalisation of oestrogen receptor- $\alpha$ within the testis and excurrent ducts of the rat and marmoset monkey from perinatal life to adulthood. J. Endocrinol., $1997 ; 153$ : 485-495.

25. FISHER J.S., TURNER K.J., FRASER H.M., SAUNDERS P.T.K., BROWN D., SHARPE R.M. Immunoexpression of aquaporin-1 in the efferent ducts of the rat and marmoset monkey during development, its modulation by estrogens, and its possible role in fluid resorption. Endocrinology, 1998 ; 139 : 3935-3945.

26. FOUCAULT P., DROSDOWSKY M.A., CARREAU S. Germ cell and Sertoli cell interactions in human testis: evidence for stimulatory and inhibitory effects. Human Reprod,, 1994 ; 9 : 2062-2068.

27. FREE M.J., JAFFE R.A. Collection of rete testis fluid from rats without previous efferent duct ligation. Biol. Reprod., $1979 ; 20: 269-278$.

28. GILL-SHARMA M.K., GOPALKRISHNAN K., BALASINOR N., PARTE P., JAYARAMAN S., JUNEJA H.S. Effects of tamoxifen on the fertility of male rats. J. Reprod. Fertil., 1993 ; 99 : 395-402.

29. GOLDZEIHER J.W., ROBERTS I.S. Identification of estrogen in the human testis. J. Clin. Endocrinol. Metab., $1952 ; 12: 143-150$.

30. GUNASEGARAM R, CHEW P.C.T., LOGANATH A., PEH K.L., RATNAM S.S. A $\Delta 4$-3-keto pathway 
for testosterone synthesis in the human spermatozoa. Arch. Androl., 1998a; 40 : 49-57.

31. GUNASEGARAM R., PEH K.L., LOGANATH A., CHEW P.C.T., RATNAM S.S., ARULKUMARAN S. Cytochrome P-450 cholesterol side chain cleavage expression in human spermatozoa. Horm. Metab. Res., 1998; 30 : 58-60.

32. HARRIS C.A., HENTTU P., PARKER M.G., SUMPTER J.P. The estrogenic activity of phthalate esters in vitro. Environ. Health Perspect., 1997; 105 : 801811.

33. HENDRY W.F., PARSLOW J.M., STEDRONSKA J. Exploratory scrotomy in 168 azoospermic males. Br. J. Urol.,1983; 55 : 785-791.

34. HESS R.A., BUNICK D., BAHR J.M. Sperm, a source of estrogen. Environ. Health Perspect., 1995; $103: 59-62$.

35. HESS R.A., GIST D.H., BUNICK D. et al. Estrogen receptor $(\alpha \& B)$ expression in the excurrent ducts of the adult male rat reproductive tract. J. Androl., $1997 ; 18: 602-611$.

36. HESS R.A., BUNICK D., LEE K H. et al. A role for estrogens in the male reproductive sytem. Nature, $1997 ; 390: 509-512$.

37. IDAOMAR M., GUERIN J.F., LORNAGE J., CZYBA J.C. Stimulation of motility and energy metabolism of spermatozoa from asthenospermic patients by 17ß-estradiol. Arch. Androl., 1989; 22 : 197-202.

38. INKSTER S., YUE W., BRODIE A. Human testicular aromatase: immunocytochemical and biochemical studies. J. Clin. Endocrinol. Metab., 1995; 80 : 19411947.

39. JANULIS L, HESS R.A., BUNICK D. et al. Mouse epididymal sperm contains active $\mathbf{P} 450$ aromatase which decreases as sperm traverse the epididymis. J. Androl., $1996 ; 17: 111-116$.

40. JANULIS L., BAHR J.M., HESS R.A, JANSEN S., OSAWA Y., BUNICK D. Rat testicular germ cells and epididymal sperm contain active P450 aromatase. J. Androl., 1998; 19: 65-71.

41. JAYLE M.F., SCHOLLER R., SFIKAKIS A., HERON M. Excrétion des phénolstéroïdes et des 17-cétostéroïdes après administration de gonadotropines à des hommes. Clin. Chim. Acta, $1962 ; 7$ : 212-20.

42. JENKINS A.D., LECHENE C.P., HOWARDS S.S. The effect of estrogen administration in vivo on the elemental composition of the intraluminal fluids of the seminiferous tubules, rete testis and epididymis of tha rat. J. Androl., 1983; $4: 272-275$.

43. KUIPER G.G.J.M., CARLSSON B., GRANDIEN K. et al . Comparison of the ligand binding specificity and transcript tissue distribution of estrogen receptors $\alpha$ and B. Endocrinology, 1997; $138: 863-870$.

44. KUIPER G.G.J.M., GUSTAFSSON J.A. The novel estrogen receptor $\beta$ subtype: potential role in the cell-and promoter-specific actions of estrogens and anti-estrogens. FEBS Lett ., 1997; $410: 87-90$.

45. KUIPER G.G.J.M., LEMMEN J.G., CARLSSON B. et al. Interaction of estrogenic chemicals and phytoestrogens with estrogen receptor $\beta$. Endocrinology 1998; 139 : 4252-4263.

46. KUMARI G.L., ALLAG I.S., DAS R.P., DATTA J.K. Regional differences in steroidogenesis and hormone levels in the epididymis and vas deferens of adult rats.Int. J. Androl., 1980; 3 : 267-281.

47. LEACH R.B., MADDOCK W.O., TOKUYAMA I., PAULSEN C.A., NELSON W.O. Clinical studies of testicular hormone production. Recent Prog. Horm. Res., 1956; 12 : 377-393.

48. LEJEUNE H., SANCHEZ P., SAEZ J.M. Enhancement of long-term testosterone secretion, and steroidogenic enzyme expression in human Leydig cells by coculture with human Sertoli cellenriched preparations. Int. J. Androl., 1998; 21 : 129 140.

49. LEVALLET J., CARREAU S. Expression in vitro du gène de l'aromatase dans les cellules testiculaires du rat. C. R. Acad. Sc. (Paris) 1997; $320: 123-129$.

50. LEVALLET J., BILINSKA B., MITTRE H., GENISSEL C., FRESNEL J., CARREAU S. Expression and immunolocalization of functional cytochrome P450 aromatase in mature rat testicular cells. Biol. Reprod., 1998 ; 58 : 919-926.

51. LEVAllet J., DELARUE B., MiTTRE H., CARREAU S. Alternative splicing events in the coding region of the $\mathrm{P} 450$ aromatase gene in male rat germ cells. J. Molec. Endocrinol., 1998 ; 20 : 305-312.

52. LI H., PAPADOPOULOS V., VIDIC B., DYM M., CULTY M. Regulation of rat testis gonocyte proliferation by platelet-derived growth factor and estradiol : identification of signaling mechanisms involved. Endocrinology, 1997; 138 : 1289-1298.

53. LUBAHN D.B., MOYER J.S., GOLDING T.S., COUSE J.F., KORACH K.S., SMITHIES O. Alteration of reproductive function but not prenatal sexual development after insertional disruption of the mouse estrogen receptor gene. Proc. Natl. Acad. Sci. USA , 1993; 90 : 11162-11166.

54. LUCONI M., BONACCORSI L., MAGGI M. et al. Identification and characterization of functional nongenomic progesterone receptors on human sperm membrane. J. Clin. Endocrinol. Metab., 1998; 83 : 877-885.

55. MARIE E., VENDRELY E, de REVIERS M., CARREAU S. Stéroides plasmatiques et intratesticulaires: existe-t-il des correlations avec la spermatogenèse chez l'homme fertile et infécond. Ann. Endocrinol., 1995; 56 : 337.

56. MARIE E., VENDRELY E, GALERAUD-DENIS I, de REVIERS M, CARREAU S. Increased testicular 
steroid concentrations in patients with idiopathic infertility and normal FSH levels. Andrologia, soumis.

57. MEISTRICH M.L., HUGUES T.J., BRUCE W.R. Alteration of epididymal sperm transport and maturation in mice by oestrogen and testosterone. Nature 1975; $258: 145-147$.

58. McCALMAN C.D., GETSIOS S., FAROOKHI R., BLASCHUK O.W. Estrogens potentiate the stimulatory effects of follicle stimulating hormone on $\mathrm{N}$ cadherin messenger ribonucleic acid levels in cultured mouse Sertoli cells. Endocrinology, 1997; $138: 41$ 49

59. Mc LACHLAN R.I., WREFORD N.G., O'DONNELL L., de KRETSER D.M., ROBERTSON D.M. The endocrine regulation of spermatogenesis: independent roles for testosterone and FSH. J. Endocrinol., $1996 ; 148: 1-9$.

60. MORISHIMA A., GRUMBACH M.M., SIMPSON E.R., FISCHER C., QIN K. Aromatase deficiency in male and female siblings caused by a novel mutation and the physiological role of estrogens. J. Clin. Endocrinol. Metab., 1995; 80 : 3689-3698.

61. MORRISH D.W., VENNER P.M., SIY O., BARRON G., BHARDWAJ D., OUTHET $\quad$ D. Mechanisms of endocrine dysfunction in patients with testicular cancer. J. Natl. Cancer Inst. 1990; 82 : 412-8.

62. MOSSELMAN S., POLMAN J., DIJKEMA R. ERß: identification and characterization of a novel human estrogen receptor. FEBS Lett., 1996; 392 : 4953.

63. NAKAZUMI H., SASANO H., MAEHARA I., OZAKI M., TEZUKA F., ORIKASA S. Estrogen metabolism and impaired spermatogenesis in germ cell tumors of the testis. J. Clin. Endocrinol. Metab., 1996; 81: 1289-1295.

64. NITTA H., BUNICK D., HESS R.A. et al. Germ cells of the mouse testis express $P 450$ aromatase. Endocrinology, 1993; 132 : 1396-1401.

65. O'MARCAIGH A., LEDGER G.A., ROCHE P.C., PARISI J.E., ZIMMERMAN D. Aromatase ex-pression in human germinomas with possible biological effects. J. Clin. Endocrinol. Metab. 1996; 80: 3763- 6 .

66. PAPADOPOULOS V,, DROSDOWSKY M.A., CARREAU S. On the existence of two Leydig cell populations in aged human testis. Ann. NY. Acad. Sci., 1987; 513 : 356-359.

67. PAYNE A.H., KELCH R.P., MUSICH S.S., HALPERN M.E. Intratesticular site of aromatization in the human. J.Clin. Endocrinol. Metab., 1976; 42 : 1081-1087.

68. PURVIS K., LANDGREN B.M., CEKAN Z., DICZFALUSY E. Indices of gonadal function in the human male. Seminal plasma levels of steroids in normal and pathological conditions. Clin.Endocrinol.,
$1975 ; 4: 247-258$.

69. REPKE K.R., MATTHES E. Tamoxifen is a $\mathrm{Na}+$ antagonistic inhibitor of $\mathrm{Na}+\mathrm{K}+$ transporting ATPase from tumor and normal cells. J. Enzyme Inhib., 1994; 8 : 207-212.

70. RAESIDE J.J. (1969) The isolation of estrone sulphate and estradiol-17ß sulphate from st a 11 i o n testis. Can. J. Biochem., 1969; 47; 811-815.

71. SAEZ J.M. Leydig cells: endocrine, paracrine and autocrine regulation. Endocr.Rev., 1994; 15 : 574-626.

72. SAUNDERS P.T.K., FISHER J.S., SHARPE R.M., MILLAR M.R. Expression of oestrogen receptor beta (ERB) occurs in multiple cell types, including some germ cells, in the rat testis. J. Endocrinol., 1998; 156 : R13-R17.

73. SHARPE R.M. Do males rely on female hormones. Nature, 1997; $390: 447-448$

74. SHARPE R.M. The roles of oestrogen in the male. TEM, $1998 ; 9: 371-377$

75. SIMPSON E.R., MAHENDROO M.S., MEANS G.D. et al. Aromatase cytochrome P450, the enzyme responsible for estrogen biosynthesis. Endocr.Rev., 1994; $15: 342-355$.

76. SIMPSON E.R., MICHAEL M.D., AGARWAL V.R., HINSHELWOOD M.M., BULUN S.E., ZHAO Y . Expression of the CYP 19 (aromatase) gene: an unusual case of alternative promoter usage. FASEB J., $1997 ; 11: 29-36$.

77. SHETTY G., KRISHNAMURTHY H., KRISHNAMURTHY H.N., BHATNAGAR S., MOUDGAL R.N. Effect of estrogen deprivation on the reproductive physiology of male and female primates. J. Steroid Biochem. Molec. Biol. 1997; 61 : 157-166.

78. SHETTY G., KRISHNAMURTHY H., KRISHNAMURTHY H.N. et al. Effect of long term treatment with aromatase inhibitor on testicular function of adult male bonnet monkeys. Steroids, 1998; 63 : 414420.

79. SMITH EP, BOYD J, FRANK GR, et al. Oestrogen resistance caused by a mutation in the oestrogen receptor gene in man. New Engl. J. Med., 1994; 331: 1056-1061.

80. SONG W.C., QIAN Y., SUN X., NEGISHI M. Cellular localization and regulation of expression of testicular estrogen sulfotransferase. Endocrinology, $1997 ; 138$; 5006-5012.

81. TASH J.S., MEANS A.R. Regulation of protein phosphorylation and motility by cyclic adenosine monophosphate. Biol. Reprod., 1982; 26 : 745-763.

82. TASH J.S., MEANS A.R. Ca2+ regulation of sperm axonemal motility. Methods Enzymol., 1987; 139 : 808-823.

83. TEA N.T., CASTANIER M., GRENIER J., SCHOLLER R. Taux d'oestrogènes dans le plasma séminal; comparaison avec le spermogramme. Ann. Endocrinol., 1972; 33 : 297-298. 
84. TONEY W.T., DANZO B.J. Developmental changes and hormonal regulation of estrogen and androgen receptors present in the rabbit epididymis. Biol. Reprod., 1988; 39 : 818-828.

85. TOPPARI J., LARSEN J.C., CHRISTIANSEN P. et al. Male reproductive health and environmental xenoestrogens. Environ.Health Perspect., 1996; 104; 741-803.

86. TSUBOTA T., NITTA H., OSAWA Y. et al. Immunolocalization of steroidogenic enzymes, P450scc, 3ßHSD, P450c17, and P450arom in the hokkaido brown bear (Ursus arctos yesoensis) testis. Gen. Comp. Endocrinol., 1993; 92 : 439-444.

87. TSUTSUMI I., FUGIMORI K., NAKAMURA R.M., MATHER J.P., ONO T., DIZEREGA G.S. Disruption of seminiferous epithelial function in the rat by ovarian protein. Biol. Reprod., 1987; 36 : 451-461.

88. van PELT A.M.M., de ROOIJ D.G., van der BURG B., van der SAAG P.T., GUSTAFSSON J.A., KUIPER G.G.J.M. Ontogeny of estrogen receptor- $\beta$ expression in rat testis. Endocrinology, 1999; $140: 478-483$.

89. VORNBERGER W., PRINS G., MUSTO N.A., SUAREZ-QUIAN C.A. Androgen receptor distribution in rat testis: new implications for androgen regulation of spermatogenesis. Endocrinology, 1994; 134 : 2307-2316.

90. YOUNG J., BULUN S.E., AGARWAL V. et al. Aromatase expression in a feminizing adrenocortical tumor. J. Clin. Endocrinol. Metab., 1996; 81: 3173-3176.

91. ZONDEK B. Mass excretion of oestrogenic hormone in the urine of the stallion. Nature, $1934 ; 193: 209$ 210 .

\author{
ABSTRACT \\ Estrogens and spermatozoa \\ I.Galeraud-Denis*, E.MARIE* , S.CARREAU
}

Aromatase is the terminal enzyme responsible for estrogen biosynthesis; it is present in the endoplasmic reticulum membrane of steroidogenic cells in vertebrates. This enzyme functions with the ubiquitous reductase as the electron donor. The aromatase gene is unique and its expression is regulated in a tissue and more precisely in a cell-specific fashion via the alternative use of several promoters located in the first exons.

This enzymatic complex is generally involved in development, reproduction, sexual differentiation and behaviour, but also in bone and lipid metabolism, brain functions and diseases such as breast and testicular tumors. The aromatase gene expression and its transduction in a fully active protein in testicular somatic cells and germ cells together with the widespread distribution of estrogen receptors $(E R \alpha \& B)$ in the testis and the genital tract of the male, are clearly in favor of a physiological role for estrogens in the spermatogenesis processings especially in sperm maturation. Therefore, we begin to understand the physiopathological roles of the estrogens in males ; indeed, the aromatase deficiency is associated with severe bone maturation problems and sterility in man. Conversely, it is also obvious that estrogens in excess are responsible of the impaired spermatogenesis. These female hormones (or the ratio androgens / estrogens) do play a physiological role in the development and maintenance of male gonadal functions and obviously, several steps are concerned especially the sperm production and maturation.

Key words: Estrogens, spermatogenesis, spermatozoa, aromatase, fertility, man. 